\title{
LISTA DE LOS MAMÍFEROS TERRESTRES DEL ESTADO DE CAMPECHE, MÉXICO
}

\author{
DANIELA GUZMÁN-SORIANO ${ }^{1}$, OSCAR G. RETANA GUIASCÓN ${ }^{1}$ \\ \& JosÉ D. CÚ-VIZCARRA ${ }^{2}$ \\ ${ }^{1}$ Laboratorio de Vida Silvestre y Colecciones Científicas (LVSCC), Centro de Estudios de Desarrollo \\ Sustentable y Aprovechamiento de la Vida Silvestre (CEDESU), Universidad Autónoma de \\ Campeche. Ciudad Universitaria Av. Agustín Melgar s/n entre Juan de la Barrera y Calle 20 Colonia \\ Buenavista C.P. 24030 Campeche, Camp., México. \\ $<$ hetigrikati@yahoo.com>y <retana1967@yahoo.com.mx> \\ ${ }^{2}$ El Colegio de la Frontera Sur, Unidad Campeche. Av. Rancho Polígono 2-A, Ciudad Industrial C.P. \\ 24500, Lerma, Campeche, Camp., México. \\ <jdvizcarra104@hotmail.com>
}

Guzmán-Soriano, D., Retana Guiascón, O. G. \& Cú-Vizcarra, J. D. 2013. Lista de los mamíferos terrestres del estado de Campeche, México. Acta Zoológica Mexicana (n. s.), 29(1): 105-128.

RESUMEN. Presentamos la lista taxonómica actualizada de los mamíferos terrestres del estado de Campeche, que se compone por 111 especies, lo que equivale al $22 \%$ de representatividad a nivel nacional; los quirópteros, carnívoros y roedores constituyen el $80 \%$ del total de los registros. De acuerdo a la distribución que siguen los mamíferos terrestres, en Campeche sólo se cuenta con un representante endémico, la subespecie Reithrodontomys gracilis insularis. En cuanto al estado de protección de las especies registradas en el Estado, se tiene que el $29 \%$ se encuentra dentro de alguna categoría de protección en la NOM-059, en tanto que la UICN anota a todas las especies, aunque el $90 \%$ en la categoría de menor importancia (LC), y por último, 21 de los registros se incluyen en los apéndices del CITES. De este modo, el presente estudio establece el estado de conocimiento de este grupo faunístico a nivel estatal y nacional, el cual es de gran utilidad en el proceso de toma decisiones para favorecer la conservación y la planificación del manejo de cada una de las especies.

Palabras Clave: Conservación, distribución, endemismo, mastofauna, Península de Yucatán.

Guzmán-Soriano, D., Retana Guiascón, O. G. \& Cú-Vizcarra, J. D. 2013. List of terrestrial mammals of the state of Campeche, Mexico. Acta Zoológica Mexicana (n. s.), 29(1): 105-128.

ABSTRACT. We present the updated taxonomic list of land mammals of the State of Campeche, which is composed of 111 species, equivalent to $22 \%$ representation at the national level; the bats, carnivores and rodents make up $80 \%$ of the records. According to the distribution of land mammals, Campeche only has a representative endemic, the subspecies Reithrodontomys gracilis insularis. Regarding the protection status of the species recorded in the state, $29 \%$ are within some category of protection in the NOM-059, while IUCN notes to all species, although 90\% in minor category (LC) and, finally, 21

Recibido: 14/12/2011; aceptado: 09/01/2013. 
records are included in the CITES Appendices. In this way, this study establishes the level of knowledge of this fauna to state and national level, which is useful in the decision making process to promote the conservation and management planning for each of the species.

Key Words: Conservation, distribution, endemism, mammalian fauna, Yucatan Peninsula.

\section{INTRODUCCIÓN}

Los mamíferos silvestres enfrentan actualmente diferentes amenazas de extinción, debido a las presiones provocadas por la fragmentación de sus hábitats, introducción de especies, cacería ilegal, entre otras, lo cual ha generado drásticas reducciones en sus áreas de distribución, provocando mayor movimiento de las poblaciones en busca de alimento o refugio (Saunders et al. 1991; Cuarón 2000). Bajo esta perspectiva, el estado de Campeche representa la última área forestal continua de selvas a nivel nacional y se incluye dentro de uno de los dos principales bloques de selva en el Neotrópico, el cual se extiende desde el Noroeste de Sudamérica a través de Centroamérica, hasta llegar al sureste mexicano, por lo que juega un papel importante como reservorio natural, situación que resulta importante, sobre todo en un país megadiverso como México (Mittermeier et al. 1997; INE 2000; Kingsolver 2003, Primack \& Corlett, 2005). El interés por conocer las especies de mamíferos que habitan en el territorio campechano, se documentó por primera vez en los trabajos de Merriam (1901 a,b) y Gaumer (1917). Sin embargo, fue hasta 1986 que se presentó la primera lista formal del componente mastofaunístico presente en la entidad (Ramírez-Pulido et al. 1986).

En la última década se han publicado distintos trabajos sobre nuevos registros de mamíferos para la Península de Yucatán, incluyendo Campeche (Hernández-Huerta et al. 2000; Escalona et al. 2002; Vargas-Contreras et al. 2002; Vargas Contreras et al. 2004; Martínez-Kú et al. 2007; Guzmán-Soriano et al. en revisión), por lo que el conocimiento de la riqueza mastofaunística para el Estado se ha incrementado satisfactoriamente. No obstante, a más de 20 años de la publicación de Ramírez-Pulido y colaboradores (1986), se carece de un documento integral y analítico en el cual se consideren los cambios nomenclaturales y taxonómicos, así como su estado de protección y afinidad geográfica. En este contexto, el objetivo del presente trabajo fue sistematizar y actualizar el conocimiento sobre la riqueza y el estado de protección de la mastofauna campechana, con la finalidad de generar información útil que contribuya a definir las especies prioritarias para su conservación.

\section{MATERIALES Y MÉTODOS}

Para la obtención de la información referente a los mamíferos terrestres de Campeche, se aplicó el método de investigación documental consultando la literatura existente en medios impresos y electrónicos, tomando como base las obras de Hall (1981) 
y Ceballos \& Oliva (2005). Asimismo, se consideraron los datos de especímenes colectados en Campeche y depositados en las colecciones mastozoológicas del Instituto de Biología de la Universidad Nacional Autónoma de México (CNMA), del Centro de Estudios de Desarrollo Sustentable de la Universidad Autónoma de Campeche (CM-UAC), las Colecciones Mastozoológicas de El Colegio de la Frontera Sur Unidad San Cristóbal (CM-SC) y Unidad Chetumal (ECOCHM) y la Colección del Instituto de Investigaciones Biológicas de la Universidad Veracruzana (IIBUV); esto con el fin de incluir las especies que tienen registro en el Estado, pero que no han sido consideradas en publicaciones formales. El arreglo taxonómico, los cambios de nomenclatura y las cifras actualizadas de los mamíferos mexicanos se determinaron basados en Ceballos et al. (2005) y Wilson \& Reeder (2005).

De acuerdo al análisis bibliográfico, se incluyen siete cambios taxonómicos. En primera instancia se considera la propuesta de Ruedas \& Salazar-Bravo (2007) para el conejo Sylvilagus brasiliensis, quienes con base en estudios morfológicos y cromosomáticos, determinan que la subespecie S. brasiliensis truei, con distribución en el Estado, sube a nivel específico como $S$. gabbi, quedando tentativamente como S. g. truei, en espera de una revisión completa de la especie. Para el caso del orden Chiroptera, se considera el cambio para el género Natalus propuesto por Tejedor (2006), quien de acuerdo a las diferencias morfológicas y los aspectos de la distribución, determina que la especie $N$. mexicanus es la que se distribuye en Campeche, sustituyendo el nombre previamente usado de $N$. stramineus que queda restringido a Sudamérica. Asimismo, Eger (2007) sugiere subir la subespecie Eumops bonariensis nannus a nivel específico como E. nanus, de acuerdo a la distribución del género para el surestre de México y quedando como especie monotípica. Un cambio que no se consideró fue el del género del Tapir (Tapirus bairdii) a Tapirella, debido a que aún existe cierta controversia entre el grupo de especialistas en cuanto a la valides de este cambio (Groves \& Grubb 2011, Medici 2011, Reyna-Hurtado, Com. pers.), por lo que se mantuvo el nombre genérico Tapirus para las poblaciones de la Península de Yucatán. Para los venados temazates se sigue la propuesta de Medellín et al. (1998) y Grubb (2005), los cuales suben a nivel de especie a Mazama americana temama, quedando sólo M. temama y M. pandora con distribución en la Península de Yucatán. Finalmente, en el orden Rodentia se consideró el cambio de género de la rata arrocera, la cual pasó de Oryzomys rostratus a Handleyomys rostratus, cambio sugerido por Musser \& Carleton (2005) y Weksler et al. (2006) en base a revisiones morfológicas y moleculares.

Para determinar la riqueza de especies presente en la entidad, se analizaron y confrontaron los datos obtenidos con respecto a las cifras publicadas para la mastofauna terrestre de México, de esta manera se procedió a comparar numérica y porcentualmente, los valores correspondientes de riqueza estatal versus riqueza nacional por nivel taxonómico (orden, familia, subfamilia, género, especie y subespecie). Cabe 
señalar que los números que se tomaron en cuenta para dichos análisis se basaron en lo reportado por Ceballos et al. (2005), pero con un detallado análisis de cada orden, puesto que se manejan irregularidades en las cifras totales que se ofrecen, y de igual manera se corroboró en literatura independiente a la mencionada obra.

La información sobre la afinidad geográfica se tomó de Ceballos \& Oliva (2005), considerando a aquellas especies que comparten distribución con Norteamérica (NA), Sudamérica (SA) y ambas (AM). La situación de endemismo a nivel Mesoamérica, México y Campeche se verificó con los trabajos de Hall (1981), Arita \& Ceballos (1997), Ceballos \& Oliva (2005) y Ceballos et al. (2005). Los datos referentes al estado de protección nacional e internacional que guardan los mamíferos de Campeche, fueron tomados de la Norma Oficial Mexicana NOM-059-SEMARNAT-2006 (Semarnat 2008), la Unión Internacional para la conservación de la Naturaleza (IUCN 2008) y el Convenio sobre el Comercio Internacional de Especies Amenazadas de Fauna y Flora Silvestres (CITES 2009).

Con lo anterior se conformó la lista taxonómica de los mamíferos terrestres de Campeche (Apéndice), en la cual, además de anotar datos sobre su afinidad geográfica, estado de protección y endemismo, se incluye información adicional relevante como clase de tamaño, gremio trófico y patrón reproductivo, tomados de Fleming (1973), Wilson (1973), Leopold (1978), Woloszyn \& Woloszyn (1982), Ceballos \& Galindo (1984), Ceballos \& Miranda (1986), Coates-Estrada \& Estrada (1986), LaVal \& Rodríguez (2002), y Sánchez-Hernández et al. (2005). De igual forma se incluye una columna donde se reporta el autor y fecha de registro de la especie en el Estado, considerando publicaciones a partir de Hall (1981). Finalmente, se agregó una columna que indica las especies representadas en la Colección Mastozoológica depositada en la Universidad Autónoma de Campeche (UAC), única institución que cuenta con una colección de este tipo en todo el Estado.

\section{RESULTADOS Y DISCUSIÓN}

Riqueza y Composición Taxonómica. Tomando en cuenta las 494 especies de mamíferos terrestres reportadas por Ceballos et al. (2005), para la República Mexicana, así como los cambios taxonómicos y registros actuales, se determina que el estado de Campeche cuenta con el $22 \%$ de esta riqueza faunística. Bajo este contexto, la mastofauna terrestre de Campeche está compuesta por 111 especies, 39 monotípicas y 72 politípicas, agrupadas en 82 géneros, 29 familias y 11 órdenes (Apéndice).

Los órdenes con mayor riqueza de especies en el Estado son: Chiroptera con 55 especies, Rodentia con 18 y Carnivora con 17, mientras que Cingulata, Soricomorpha y Perissodactyla sólo cuentan con una especie cada uno (Cuadro 1). Esto es lo esperado, si se considera que en el país únicamente se registran dos especies del 


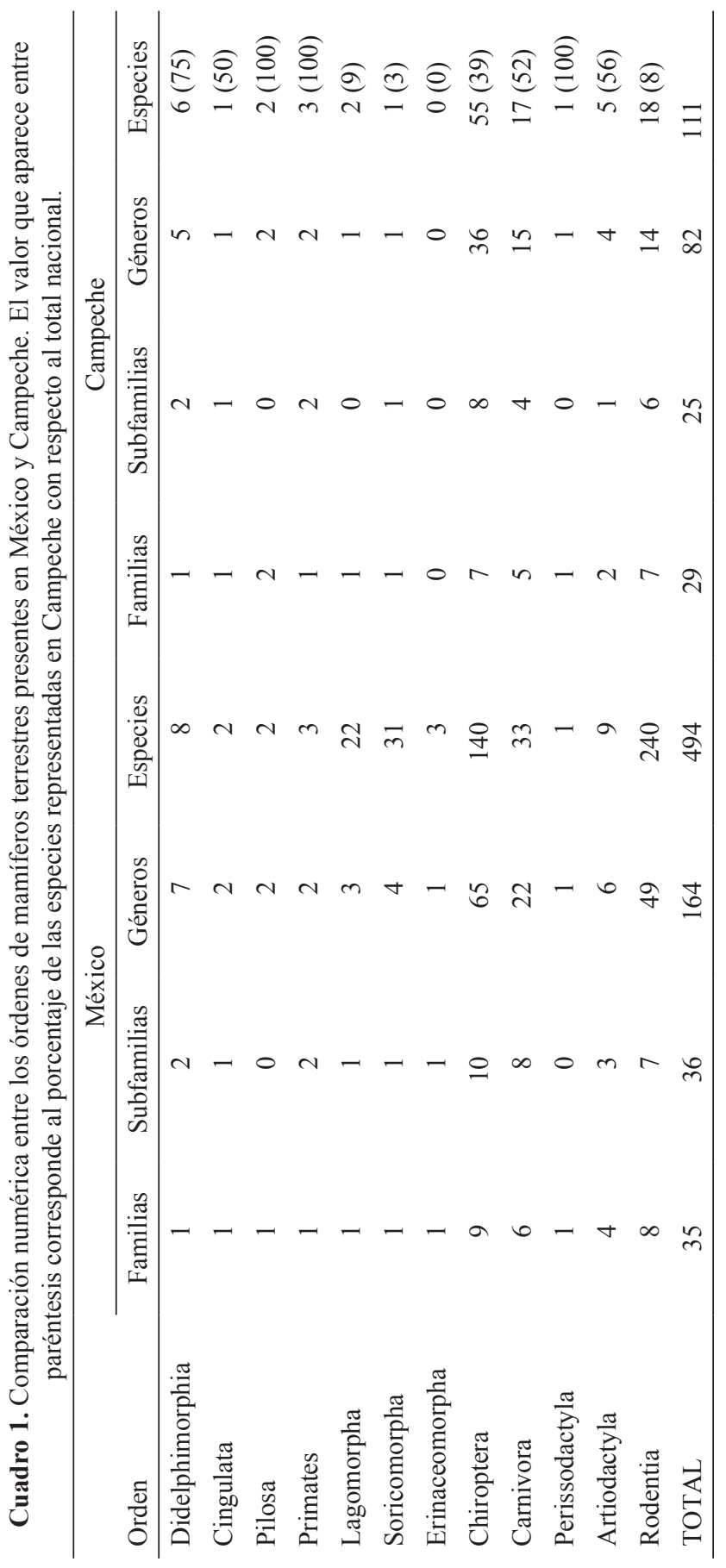


orden Cingulata, Dasypus novemcinctus y Cabassous centralis y un solo perisodáctilo, T. bairdii conocido en Campeche como Tzimin. Un caso contrario es el orden Soricomorpha, ya que de las 31 especies de musarañas enlistadas para México sólo se reporta Criptotys mayensis para el Estado, conocida comúnmente como musaraña maya (Ceballos et al. 2005).

Los 55 quirópteros registrados en la entidad constituyen el 39\% de la riqueza de especies del total que habita en el territorio mexicano (140). Se agrupan en 36 géneros y siete familias, siendo la familia Phyllostomidae, con 27 especies, la mejor representada, contando con cinco de las ocho subfamilias reportadas para el país (Fig. 1). De la subfamilia Desmodontinae, compuesta únicamente por tres especies de hábitos hematófagos, dos habitan en Campeche (Diphylla ecaudata y Desmodus rotundus). De la subfamilia Phyllostominae destaca para el estado la presencia de la especie Vampyrum spectrum, considerado el murciélago más grande de América y un depredador importante en la cadena trófica. Por otra parte, en la entidad se cuenta con el $100 \%$ de representación de la familia Mormoopidae a nivel nacional.

A pesar de que el orden Rodentia es el segundo de mayor riqueza a nivel estatal, sólo se tiene representado el $8 \%$ del total nacional, pues únicamente se registran 18 especies agrupadas en 14 géneros y siete familias; de estas últimas la familia Cricetidae es la mejor representada con nueve especies (Fig. 2). Estos valores son los

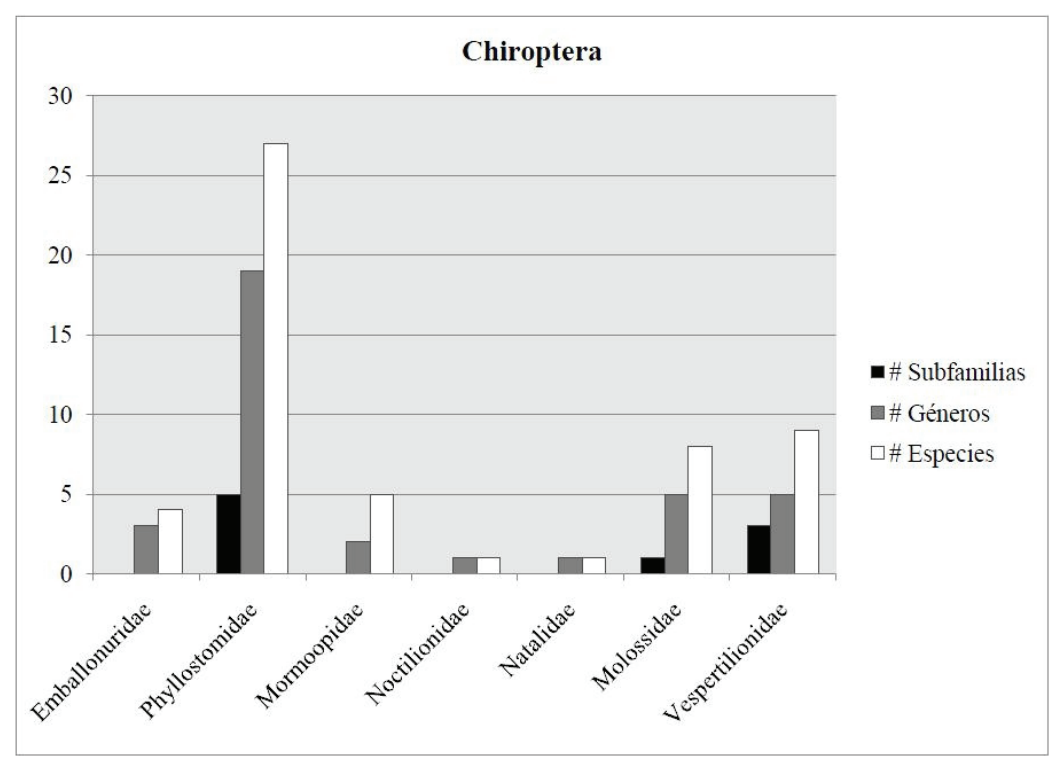

Figura 1. Composición del orden Chiroptera en el Estado de Campeche por familia. 


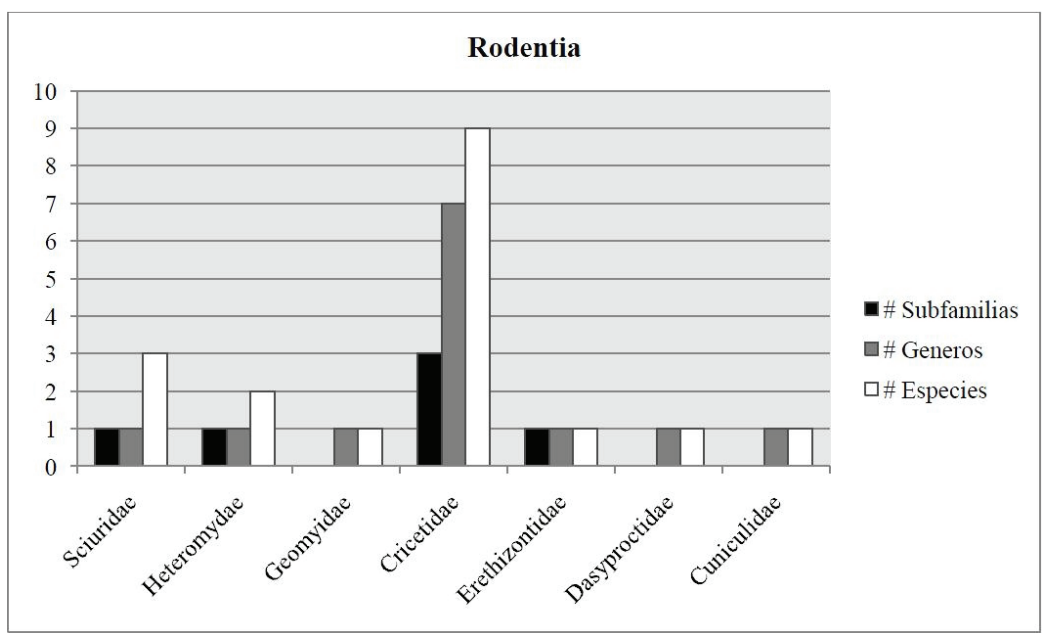

Figura 2. Composición taxonómica por familia para el orden Rodentia en el Estado de Campeche.

esperados para regiones con poca heterogeneidad fisiográfica y ecológica como lo es la Península de Yucatán (Arita \& Ceballos 1997).

En Campeche se cuenta con el 52\% de los mamíferos carnívoros que habitan en nuestro país, las 17 especies anotadas para el Estado pertenecen a 15 géneros y cinco de las seis familias reconocidas a nivel nacional, de las cuales Felidae se encuentra representada por cinco de las seis especies de gatos silvestres reportadas para México: Puma concolor, P. yagouaroundi, Leopardus pardalis, L. wiedii y Panthera onca. Estos valores son de destacarse, dada la importancia que tiene este orden como indicador en la salud de un hábitat y de las poblaciones de sus presas (Ceballos et al. 2002; Medellín et al. 2002).

Los órdenes Pilosa, Primates y Perissodactyla están representados al 100\% en el territorio campechano, aunque cabe señalar que estos taxa no son muy ricos a nivel nacional, ya que para Pilosa sólo se reportan dos especies de oso hormiguero (Tamandua mexicana y Cyclopes didactylus). Hay tres especies de Primates: el mono araña (Ateles geoffroyi) y dos especies de mono aullador (Alouatta palliata y A. pigra), mientras que para Perissodactyla sólo se tiene a T. bairdii.

Por otro lado, de las ocho especies de marsupiales (orden Didelphimorphia) que habitan en México, siete se distribuyen en Campeche. En cuanto al orden Lagomorpha, sólo dos especies de conejos pertenecientes al género Sylvilagus se localizan en territorio campechano, constituyendo el $9 \%$ del total de conejos y liebres reportadas para el territorio nacional. Para el orden Artiodactyla se cuenta con una representación del $56 \%$ de las especies de ungulados registrados para la República Mexicana, 
contando con dos de las cuatro familias y cuatro de los siete géneros que componen a este orden.

Distribución y Endemismo. La distribución de la fauna mexicana está influenciada por la combinación de elementos neárticos y neotropicales, debido a que en el territorio mexicano confluyen ambas regiones zoogeográficas. Esta situación también es patente para el caso de los mamíferos terrestres registrados en Campeche. De los mamíferos que no presentan alguna condición de endemismo, el 56\% de las especies tienen una filiación neotropical, por lo que su distribución se extiende hacia Sudamérica (SA), entre los que se encuentran tres marsupiales, los dos representantes del orden Pilosa, el mono aullador (A. palliata), el conejo de monte ( $S$. gabbi), 43 murciélagos, cinco carnívoros, el pecarí de labios blancos (Tayassu pecari), el tapir (T. bairdii) y, cuatro roedores.

El 25\% de los mamíferos campechanos tienen un patrón de distribución neártica y neotropical (AM), entre los que se incluyen el tlacuache común (Didelphis virginiana), el armadillo de nueve bandas ( $D$. novemcinctus), el conejo de monte ( $S$. floridanus), ocho especies del orden Chiroptera, nueve carnívoros en donde se incluyen los cinco felinos reportados, así como dos artiodáctilos y dos roedores. Solamente el 4\% de los mamíferos registrados en Campeche tienen una afinidad meramente neártica (NA), estos son: el murciélago vespertiliónido Lasiurus intermedius, los carnívoros Canis latrans y Spilogale angustifrons y el ratón cricétido Peromyscus leucopus (Fig. 3).

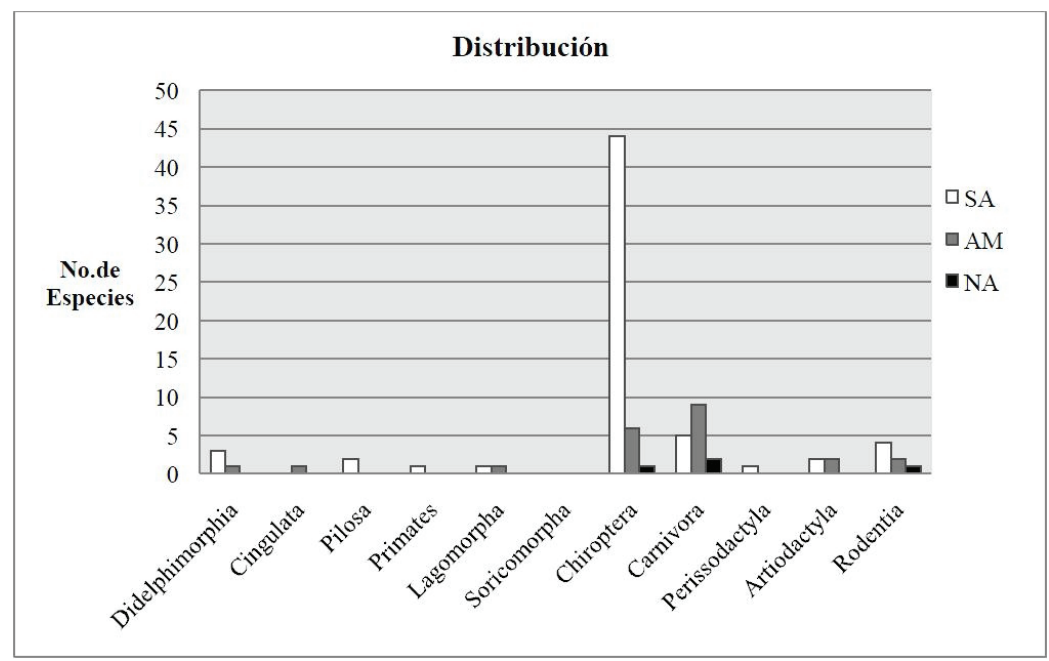

Figura 3. Representatividad de la distribución en los órdenes registrados para Campeche: Sudamérica (SA), Norteamérica (NA) y en ambas (AM). 
En las regiones tropicales con una reciente historia geológica y sin grandes complejos orográficos, como la Península de Yucatán, se registran pocas especies endémicas. Como parte de esto, en el estado de Campeche únicamente se reporta la subespecie de roedor Reithrodontomys gracilis insularis como endémica, la cual fue registrada en la isla del Carmen por Jones (1964) y de la cual habría que evaluar su estado de conservación (Arita et al.1997, Ceballos et al. 1998). En lo que respecta a los mamíferos catalogados como endémicos de México, y cuya área de distribución comprende el estado de Campeche en su totalidad o parte de éste, se encuentran el marsupial Tlacuatzin canescens, que habita en la región norte de Campeche y el territorio del estado de Yucatán; y el roedor Peromyscus yucatanicus, restringido al territorio mexicano de la Península de Yucatán.

Para el caso de los mamíferos que se califican como endémicos a Mesoamérica y cuya área de distribución comprende el territorio campechano, se ubican 20 especies: un marsupial (Marmosa mexicana), la musaraña maya (C. mayensis); el cacomiztle (Bassariscus sumichrasti), el venado temazate ( $M$. pandora), 10 roedores, cuatro murciélagos, el mono araña (A. geoffroyi) y el aullador negro (A. pigra; Fig. 4). Cabe destacar que la ardilla yucateca (Sciurus yucatanensis), los ratones Heteromys gaumeri y Otonyctomys hatti; la musaraña maya (C. mayensis) y el venado temazate (M. pandora), están restringidos a la Península de Yucatán, abarcando parte de Belice y Guatemala.

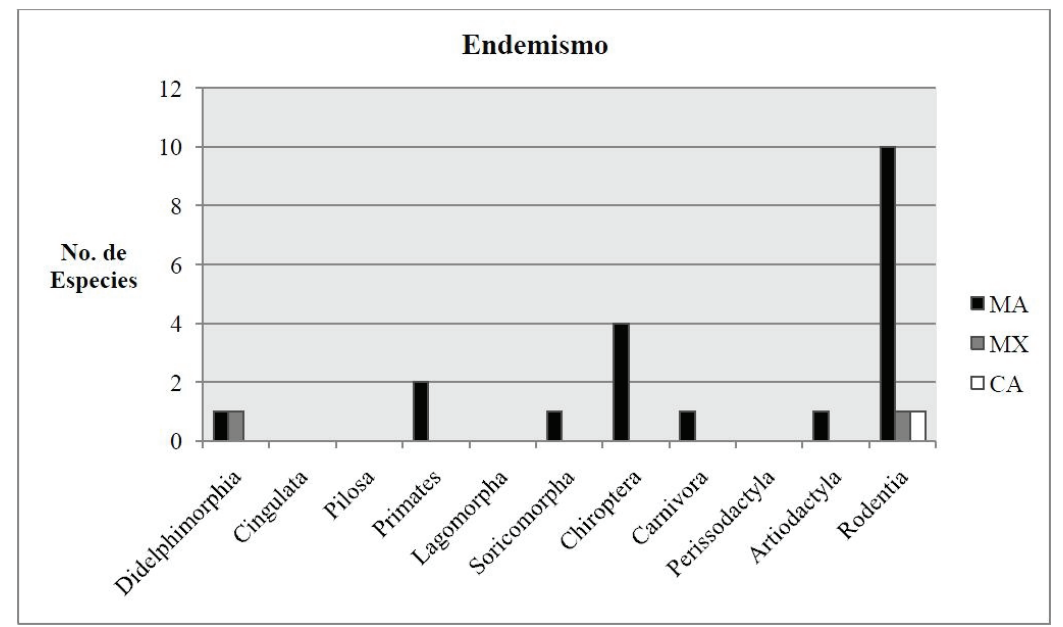

Figura 4. Representatividad de los órdenes de mamíferos registrados que presentan algún grado de endemismo. MA: Endémicos a Mesoamérica, MX: Endémicos a México y CA: Endémicos a Campeche. 
Estado de Conservación. De acuerdo a la NOM-059-SEMARNAT-2006 (Semarnat 2008), 31 especies y cinco subespecies de la mastofauna campechana ostentan estado de protección; 12 de las cuales se califican en peligro (P), como la subespecie de oso hormiguero (T. m. mexicana) y las dos especies de primates. En la categoría de amenazadas (A), se enlistan 13 especies y la subespecie endémica de ratón $(R . g$. insularis), así como el ratón endémico de la Península de Yucatán $(O$. hatti). Siete especies y 10 subespecies se catalogan con protección especial (Pr), destacando la presencia de la musaraña maya (C. mayensis), restringida a la Península de Yucatán (Cuadro 2).

A nivel internacional, dentro de la lista roja elaborada por la Unión Internacional para la Conservación de la Naturaleza (IUCN por sus siglas en inglés), se encuentran las 111 especies de la mastofauna campechana; no obstante, el 91\% de éstas (100 especies) se incluyen en la categoría de menor importancia (LC), la cual se caracteriza por incluir taxa abundantes y de amplia distribución. Solamente la subespecie del mono araña (A. g. yucatanensis), junto con el mono aullador (A. pigra) y el tapir ( $T$. bairdii) se catalogan en peligro (EN), en tanto el venado temazate ( $M$. pandora) se encuentra como vulnerable (VU). Para el caso de la nutria (Lontra longicaudis) y el venado cabrito (M. temama) no se cuentan con datos suficientes para su evaluación (DD); en tanto cinco especies están casi amenazadas (NT).

En los apéndices del Convenio Internacional de Tráfico de Especies Silvestres (CITES), se incluyen 21 especies de mamíferos presentes en Campeche. En el apéndice I se anotan el mono aullador, cinco carnívoros (cuatro de ellos felinos); el tapir

Cuadro 2. Número de especies protegidas y categoría de protección. Los números en paréntesis representan las subespecies.

\begin{tabular}{lccccccccccc}
\hline & \multicolumn{3}{c}{ NOM-059 } & \multicolumn{3}{c}{ IUCN } & \multicolumn{3}{c}{ CITES } \\
\cline { 2 - 10 } \multicolumn{1}{c}{ Orden } & P & A & PR & EN & VU & NT & LC & DD & I & II & III \\
\hline Didelphimorphia & - & - & 1 & - & - & - & 6 & - & - & - & - \\
Cingulata & - & - & - & - & - & - & 1 & - & - & - & - \\
Pilosa & $1(1)$ & - & - & - & - & - & 2 & - & - & - & 1 \\
Primates & 3 & - & - & $2(1)$ & - & - & 1 & - & 2 & 1 & - \\
Lagomorpha & - & - & - & - & - & - & 2 & - & - & - & - \\
Soricomorpha & - & - & 1 & - & - & - & 1 & - & - & - & - \\
Chiroptera & 1 & 8 & $4(1)$ & - & - & 2 & 53 & - & - & - & - \\
Carnivora & 4 & 3 & $2(1)$ & - & - & 2 & 14 & 1 & 5 & 1 & 5 \\
Perissodactyla & 1 & - & - & 1 & - & - & - & - & 1 & - & - \\
Artiodactyla & 1 & - & - & - & 1 & 1 & 2 & 1 & - & 1 & - \\
Rodentia & - & $2(1)$ & - & - & - & - & 18 & - & 1 & - & 3 \\
\hline
\end{tabular}


y el puerco espín (Sphiggurus mexicanus). En el apéndice II se encuentran el mono araña (A. geoffroyi), el puma ( $P$. concolor) y el pecarí de labios blancos ( $T$. pecari); por último, en el apéndice III se catalogan nueve especies: el oso hormiguero ( $T$. mexicana), cinco carnívoros y tres especies de roedores (Apéndice, Cuadro 2).

El presente trabajo constituye el primer diagnóstico en la entidad para el proceso de toma de decisiones respecto a la instrumentación de las estrategias que favorezcan el uso sustentable de este recurso natural a nivel local y regional, y por ende, su conservación a largo plazo. Además, es pertinente llevar a cabo estudios sobre la posibilidad de crear corredores que favorezcan la conectividad entre áreas tanto dentro como fuera del Estado, de tal modo que se garantice la diversidad genética y la disminución del aislamiento de especies por efecto de la fragmentación y la consecuente pérdida del hábitat, situación que amenaza especies de importancia ecológica y económica en proporciones desconocidas en la actualidad.

\section{CONCLUSIONES}

En suma, la mastofauna terrestre de Campeche está representada por 111 especies, lo que equivale al $22 \%$ de la riqueza total registrada para nuestro país. Los quirópteros, carnívoros y roedores constituyen el $80 \%$ del componente mastofaunístico de la entidad.

La lista de mamíferos anotada en este trabajo no es definitiva, ya que distintos autores coinciden que otras especies se consideran potenciales para el estado, pero aún no se cuenta con su registro de colecta, por lo que es necesario conducir muestreos en los sitios aún no explorados.

La conservación de los mamíferos de México es una prioridad nacional, en este sentido, los estudios tendientes a establecer el estado de conocimiento de este grupo faunístico a nivel estatal y nacional son de gran utilidad en el proceso de toma decisiones para favorecer, no sólo la conservación de las especies y sus hábitats, sino además la de los bienes y servicios ecológicos, económicos y sociales que sus poblaciones brindan.

\section{LITERATURA CITADA}

Arita, H. T. \& Ceballos, G. 1997. Los Mamíferos de México: Distribución y Estado de Conservación. Revista Mexicana de Mastozoología, 2: 33-71.

Ceballos, G., Medellin, R. A. \& Rodríguez, P. 1998. Assessing conservation priorites in megadiverse Mexico. Mammalian diversity, endemicity and endangerment. Ecological Aplication, 8: 8-17.

Ceballos, G., Arroyo-Cabrales, J. \& Medellín, R. A. 2002. Mamíferos de México, pp. 377-413. In: Ceballos, G. \& Simonetti, J. A. (Eds.). Diversidad y Conservación de los Mamíferos Neotropicales. CONABIO-UNAM. México, D.F.

Ceballos, G., Arroyo-Cabrales, J., Medellín, R. A. \& Domínguez-Castellanos, Y. 2005. Lista Actualizada de los Mamíferos de México. Revista Mexicana de Mastozoología. 9:21-27. 
Ceballos, G., Chávez, C., Rivera, A., Manterola, C. \& Wall, B. 2002. Tamaño Poblacional y Conservación del Jaguar en la Reserva de la Biosfera de Calakmul, Campeche, México, pp. 403-417. In: Medellín, R.A., Equihua, C., Chetkiewcz, C. L. B., Crawshaw Jr. P.G., Rabinowitz, A., Redford, K. H., Sanderson, E. W. \& Taber, A. B. (Comps.). El Jaguar en el Nuevo Milenio. Fondo de Cultura Económica, Universidad Nacional Autónoma de México y Wildlife Conservation Society. México.

Ceballos, G. \& Miranda, A. 1986. Los mamíferos de Chamela, Jalisco. Instituto de Biología, Universidad Nacional Autónoma de México. México, D.F.

Ceballos, G. \& Galindo, C. 1984. Los mamíferos de la cuenca de México. Limusa, México, D.F.

Ceballos, G. \& Oliva, G. 2005. Los mamiferos silvestres de México. CONABIO - Fondo de Cultura Económica, México, D.F.

Coates-Estrada, R. \& Estrada, A. 1986. Manual de identificación de campo de los mamíferos de la estación de Biología "Los Tuxtlas". Instituto de Biología, Universidad Nacional Autónoma de México. México, D.F.

Convention on International Trade in Endangered Species of Wild Fauna and Flora (CITES). 2009. Appendices I, II and III. UNEP.

Cuarón, A. D. 2000. Effects of land-cover changes on mammals in a neotropical region: a modeling approach. Conservation Biology, 14: 1676-1692.

Eger, J. L. 2007. Family Molossidae, pp. 399-440. In: Gardner, A. L. (Ed). Mammals of South America. University Chicago Press, Chicago.

Escalona, G., Vargas-Contreras, J.A. \& Interián-Sosa, L. 2002. Registros Importantes de mamíferos para Campeche. Revista Mexicana de Mastozoología, 6: 166-170.

Fleming, T. H. 1973. Numbers of mammal species in North and Central American forest communities. Ecology, 55: 555-563.

Gallo, J. P. 1997. Situación y Distribución de las Nutrias en México, con Énfasis en Lontra longicaudis annectens Major, 1897. Revista Mexicana de Mastozoología, 2: 10-32.

García, M. 2003. Papel ecológico de las aguadas para murciélagos insectívoros en un bosque tropical subhúmedo. Tesis (Maestría en Ciencias) El Colegio de la Frontera Sur-Chetumal. 39 pp.

Gaumer, G. F. 1917. Monografía de los mamíferos de Yucatán. Departamento de Talleres Gráficos de la Secretaría de Fomento, México, xii +331 pp.

Groves, C. \& Grubb, P. 2011. Ungulate Taxonomy. The Johns Hopkins University Press, Baltimore.

Grubb, P. 2005. Orders Perissodactyla and Artiodactyla, pp. 629-722, In: Wilson, D.E. \& Reeder, D.M. (Eds.). 2005. Mammal Species of the World. Johns Hopkins University Press.

Guzmán-Soriano, D., Vargas-Contreras, J. A., Cú-Vizcarra, J. D., Escalona Segura, G., Retana Guiascón, O. G., González Christen, A., Benítez Torres, J. A., Arroyo-Cabrales, J., Puc Cabrera, J. C. \& Victoria Chán, E. 2013. Registros Notables de Mamíferos para Campeche, México. Acta Zoológica Mexicana (n. s.), 29(2): en prensa.

Hall, E. R. 1981. The Mammals of North America. John Wiley, New York.

Hernández-Huerta, A., Sosa, V. J., Aranda, J. M. \& Bello, J. 2000. Noteworthy records of small mammals from the Calakmul biosphere reserve in the Yucatán Peninsula, Mexico. The Southwestern Naturalist, 45: 340-344.

INE (Instituto Nacional de Ecología). 2000. Programa de Manejo de la Reserva de la Biosfera Calakmul. México. 277 pp.

IUCN 2008. IUCN Red List of Threatened Species. Version 2009.2. <www.iucnredlist.org>. (Downloaded on 18 January 2010).

Jones, J. K., Jr. 1964. A new subspecies of harvest mouse, Reithrodontomys gracilis, from Isla del Carmen, Campeche. Proceedings of the Biology Society of Washington, 77: 123-124.

Kingsolver, B. 2003. The way to new vida. Sierra (San Francisco), 88: 5-34. 
LaVal, R. K. \& Rodríguez-H, B. 2002. Murciélagos de Costa Rica: Bats. Instituto Nacional de Biodiversidad (INBio). Costa Rica.

Leopold, A. S. 1978. Fauna Silvestre de México. Pax México, D.F.

López-Wilchis, R. \& López Jardines, J. 1998. Los Mamíferos de México depositados en Colecciones de Estados Unidos y Canadá. Vol. 1. Universidad Autónoma Metropolitana Unidad Iztapalapa.

Lorenzo, C., Espinosa, E. E., Naranjo, E. J. \& Bolaños, J. E. 2008. Mamíferos terrestres de la frontera sur de México, pp. 147-164. In: Lorenzo, C., Espinoza, E. \& Ortega, J. (Eds). Avances en el Estudio de los Mamíferos de México II. Asociación Mexicana de Mastozoología A.C. México.

Mac-Swiney, M.C., Sosa Escalante, J. \& Selem-Salas, C. 2003. Ampliación en la distribución de Eumops underwoodi Goodwin, 1940 (Chiroptera: Molossidae) en la Península de Yucatán, México. Revista Mexicana de Mastozoología, 7: 55-57.

Martínez-Kú, D.H., Escalona-Segura, G. \& Vargas-Contreras, J. A. 2007. Primer Registro del Zorrillo Manchado del Sur Spilogale angustifrons Howell 1902 para el Estado de Campeche, México. Acta Zoológica Mexicana, 23: 175-177.

Martínez-Kú, D.H., Escalona-Segura, G. \& Vargas-Contreras, J. A. 2008. Importancia de las Aguadas para los Mamíferos de talla Mediana y Grande en Calakmul, Campeche, México, pp. 449-468 In: Lorenzo, C., Espinoza, E. \& Ortega, J. (Eds). Avances en el Estudio de los Mamíferos de México II. Asociación Mexicana de Mastozoología A.C. México.

Medellín, R. A., Gardner, A. L. \& Aranda, J. M. 1998. The taxonomic status of the Yucatán brow brocket, Mazama pandora (Mammalia: Cervidae). Proceedings of the Biology Society of Washington, 111: 1-14.

Medellín, R.A., Equihua, C., Chetkiewics, C., Rabinowitz, A., Crawshaw, P., Redford, K., Robinson, J. G., Sanderson, E. \& Taber, A. (Eds.). 2002. El jaguar en el nuevo milenio. Fondo de Cultura Económica, Universidad Nacional Autónoma de México y Wildlife Conservation Society, México D.F.

Medici, E. P. 2011. Family Tapiridae (tapirs), pp. 182-204. In: Wilson, D. E. \& Mittermeier, R. A. (Eds). Handbook of the mammals of the world. Vol. 2. Hoofed mammals. Lynx Edicions, Barcelona.

Merriam, C. H. 1901a. Sixth New Mammal for Cozumel Island, Yucatan. Proceedings of the Biology Society of Washington, 14: 99-104.

Merriam, C. H. 1901b. Descriptions of four new pecaries from Mexico. Proceedings of the Biology Society of Washington, 14: 119-124.

Mittermeier, R. A., Mittermeier, C. G. \& Robles Gil, P. 1997. Megadiversidad, los países biológicamente más ricos del mundo. CEMEX, México.

Musser, G. G., \& Carleton, M. D. 2005. Superfamily Muroidea, pp. 894-1532. In: Wilson, D.E. \& Reeder, D. M. (Eds). 2005. Mammal Species of the World. Johns Hopkins University Press.

Navarro E., Pozo, C. \& Escobedo, E. 2003. Afinidad ecológica y distribución actual de Primates (Cebidae) en Campeche, México. Revista de Biología Tropical, 51: 591-600.

Primack, R. B. \& Corlett, R. T. 2005. Tropical Rain Forests: An Ecological and Biogeographical Comparison. Blackwell Science, Oxford.

Ramírez-Pulido, J., Britton, M. C., Perdomo, A. \& Castro, A. 1986. Guía de los Mamíferos de México, Referencias hasta 1983. Universidad Autónoma Metropolitana Unidad Iztapalapa.

Ruedas, L. A. \& Salazar-Bravo, J. 2007. Morphological and chromosomal taxonomic assessment of Sylvilagus brasiliensis gabbi (Leporidae). Mammalia, 71: 63-69.

Sánchez-Hernández., C. \& Romero, M. L. 1995. Murciélagos de Tabasco y Campeche: una propuesta para su conservación. CUADERNOS 24. Instituto de Biología, Universidad Nacional Autónoma de México. 
Sánchez-Hernández, C., Romero-Almaraz, M. L. \& García-Estrada, C. 2005. Mamíferos, pp. 283304. In: Bueno, J., Álvarez, F. \& Santiago, S. (Eds). Biodiversidad del Estado de Tabasco. Instituto de Biología. UNAM-CONABIO. México.

Saunders, D. A., Hobbs, R. J. \& Margules, C. R. 1991. Biological consequences of ecosystem fragmentation: a review. Conservation Biology, 5: 18-32.

Secretaria de Medio Ambiente y Recursos Naturales (SEMARNAT). 2006. Programa de Conservación y Manejo de la Reserva de la Biósfera Los Petenes. CONANP-SEMARNAT. 203 pp.

Secretaria de Medio Ambiente y Recursos Naturales (SEMARNAT). 2008. Norma Oficial Mexicana PROY-NOM-059-SEMARNAT-2006. Protección ambiental-especies nativas de México de flora y fauna silvestres-categorías de riesgo y especificaciones para su inclusión, exclusión o cambio-lista de especies en riesgo. Diario Oficial de la Federación, Segunda Sección, diciembre 2008.

Tejedor, A. 2006. The type locality of Natalus stramineus (Chiroptera: Natalidae): implications for the taxonomy and biogeography of the genus Natalus. Acta Chiropterologica, 8: 361-380.

Vargas-Contreras, J. A. 2008. Relación de la Complejidad del Hábitat con la Diversidad de Murciélagos en Campeche, México. Tesis de Doctorado. Universidad Nacional Autónoma de México. 134 pp.

Vargas-Contreras, J. A., Escalona-Segura, G., Cú-Vizcarra, J. D., Arroyo-Cabrales, J. \& Medellín, R. A. 2008. Estructura y diversidad de los ensambles de murciélagos en el centro y sur de Campeche, México, pp. 551-577. In: Lorenzo, C., Espinoza, E. \& Ortega, J. (Eds). Avances en el Estudio de los Mamíferos de México II. Asociación Mexicana de Mastozoología A.C. México.

Vargas-Contreras, J. A., Arroyo-Cabrales, J., Sanvicente-López, M. \& Reyna-Hurtado, R. 2002. Nuevos registros de mamíferos para Campeche. Vertebrata Mexicana, 11: 17-20.

Vargas-Contreras, J. A., Herrera-Herrera, J. R. \& Escobedo-Cabrera, J. E. 2004. Noteworthy records of mammals from Campeche, México. Revista Mexicana de Mastozoología, 8: 61-69.

Weksler, M., Percequillo, A. R. \& Voss, R. S. 2006. Ten new genera of Oryzomyine rodents. American Museum. Novitates, 3537: 1-29.

Wilson, D. E. 1973. Bat Faunas: A trophic comparison. Systematic Zoology, 22: 14-29.

Wilson, D. E. \& Reeder, D. M. (Eds). 2005. Mammal Species of the World. Johns Hopkins University Press. 2142 pp.

Woloszyn, D. \& Woloszyn, B. W. 1982. Los Mamíferos de la Sierra de la Laguna, Baja California Sur. Consejo Nacional de Ciencia y Tecnología, México, D.F. 


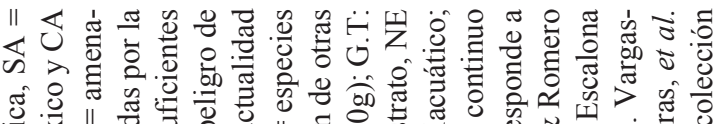

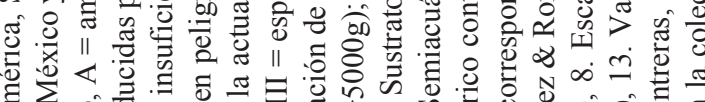

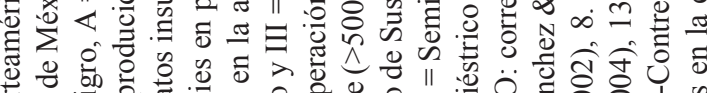
Z ठี

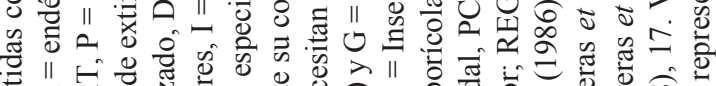

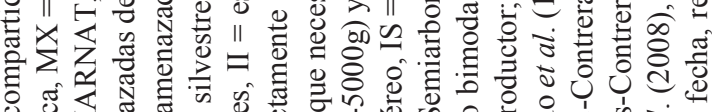

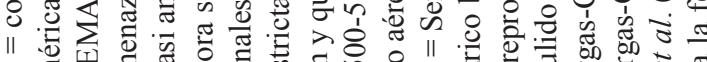

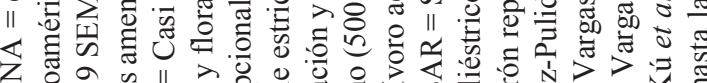

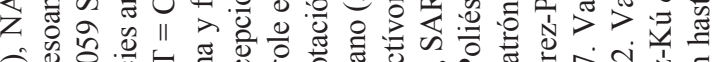

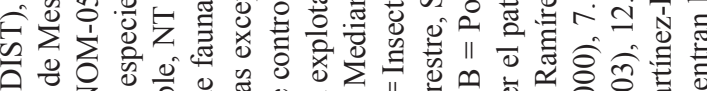

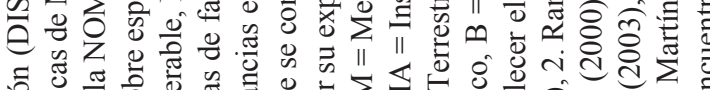

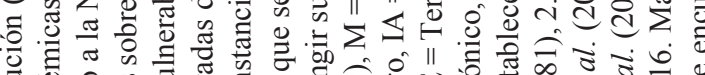

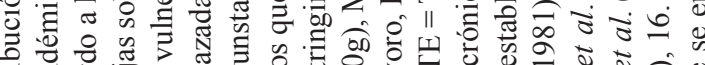

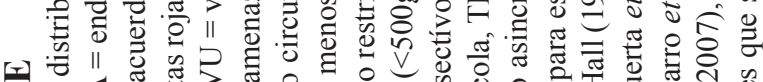
닌

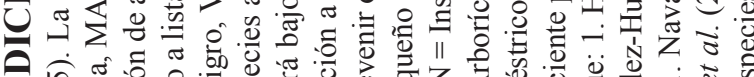

Z穴.

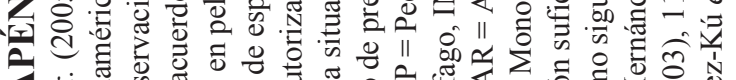

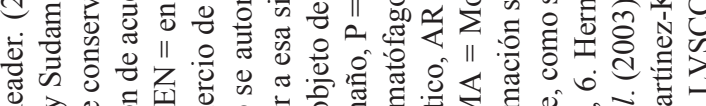
๙ め)

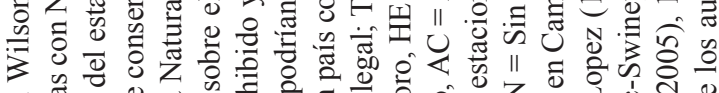

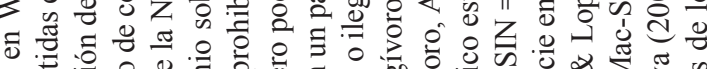

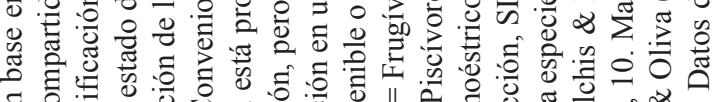

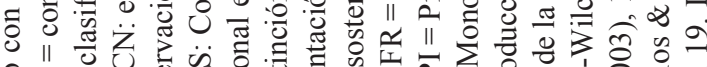

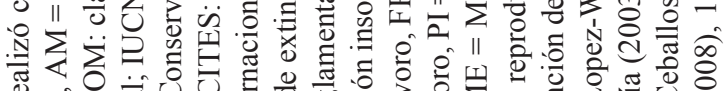

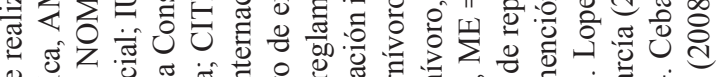
क :

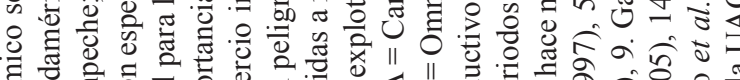

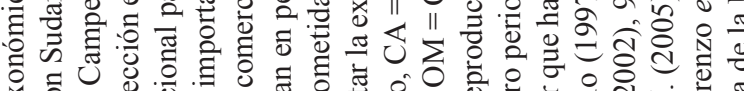

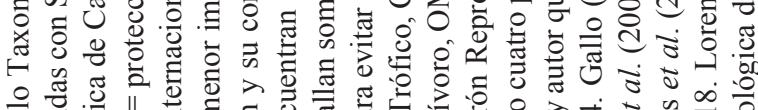
을 疍

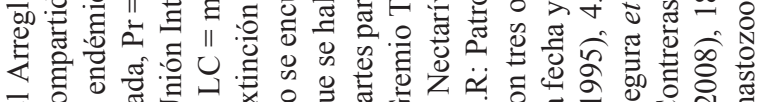

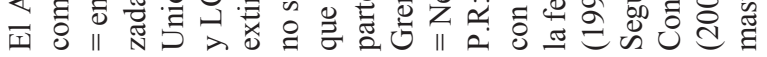
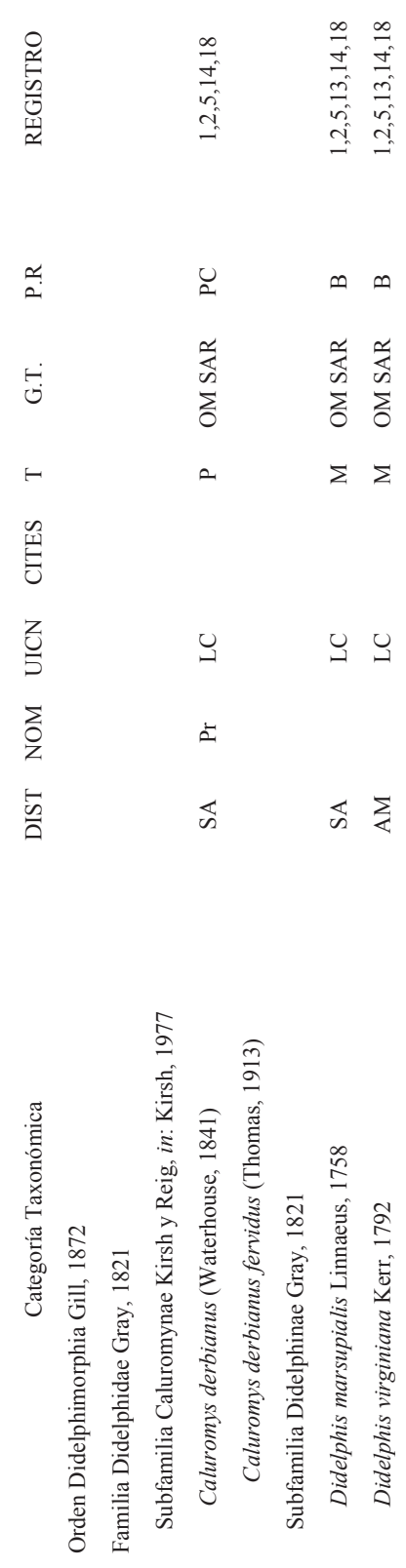


\begin{tabular}{|c|c|c|c|c|c|c|c|c|}
\hline & & : & : & & : & & : & : \\
\hline$\stackrel{\infty}{\circ}$ & 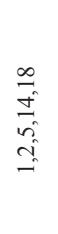 & $\begin{array}{l}\stackrel{\infty}{\simeq} \\
\stackrel{(}{\simeq}\end{array}$ & 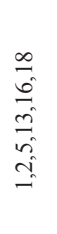 & $\begin{array}{l}\frac{\infty}{ \pm} \\
\underset{f}{f}\end{array}$ & 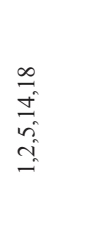 & 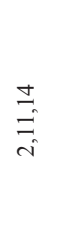 & 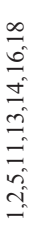 & 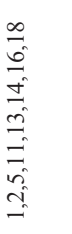 \\
\hline$\Sigma$ & $\infty$ & O & $\stackrel{\amalg}{\Sigma}$ & $\infty$ & $\mathbb{\Sigma}$ & 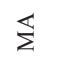 & 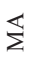 & 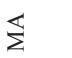 \\
\hline$\sum_{0}^{\infty}$ & $\begin{array}{l}\stackrel{\sharp}{4} \\
\sum\end{array}$ & 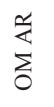 & $\begin{array}{l}\text { 菂 } \\
\text { z }\end{array}$ & $\begin{array}{l}\stackrel{a}{<} \\
z\end{array}$ & $\begin{array}{l}\stackrel{\approx}{<} \\
z\end{array}$ & & & \\
\hline a & $a$ & $a$ & $\Sigma$ & $a$ & $\Sigma$ & 0 & 0 & $\Sigma$ \\
\hline & & & & & $\exists$ & - & - & $=$ \\
\hline U્ય & u & U & $\underset{\Theta}{\cup}$ & ن. & U & u & 奋 & 鿬奋 \\
\hline & & & & $a$ & $a$ & $a$ & $a$ & $a$ \\
\hline$\Sigma$ & 芯 & 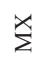 & $\sum$ & 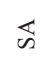 & 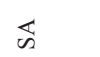 & $\overleftrightarrow{n}$ & $\$$ & 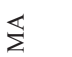 \\
\hline
\end{tabular}

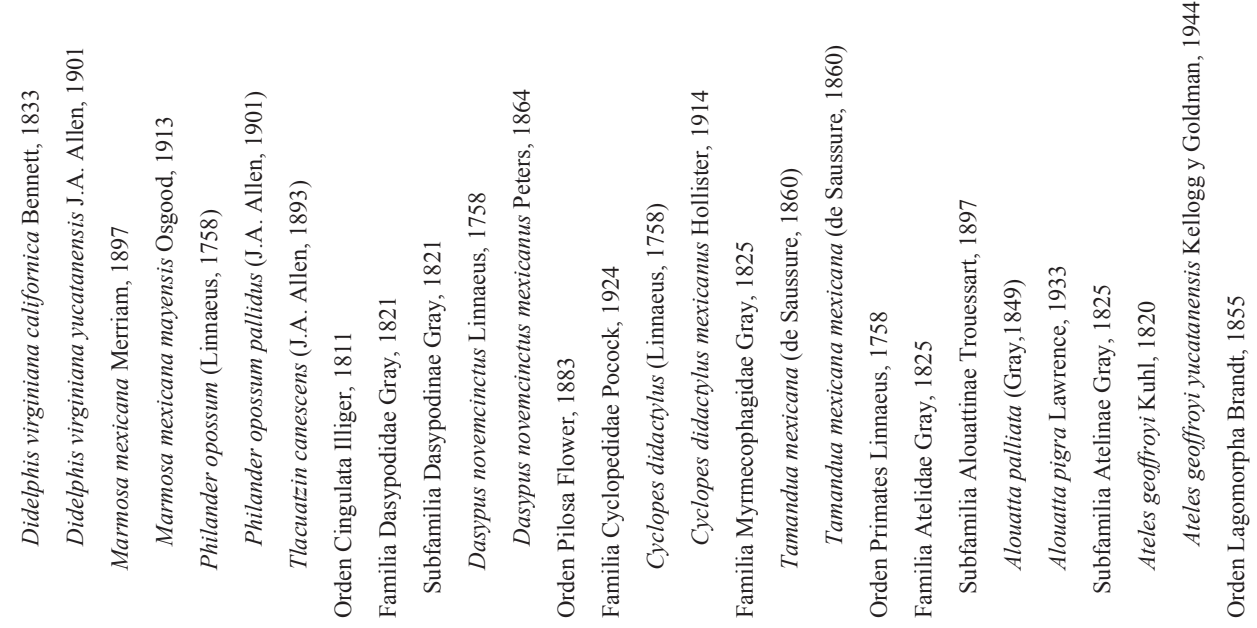




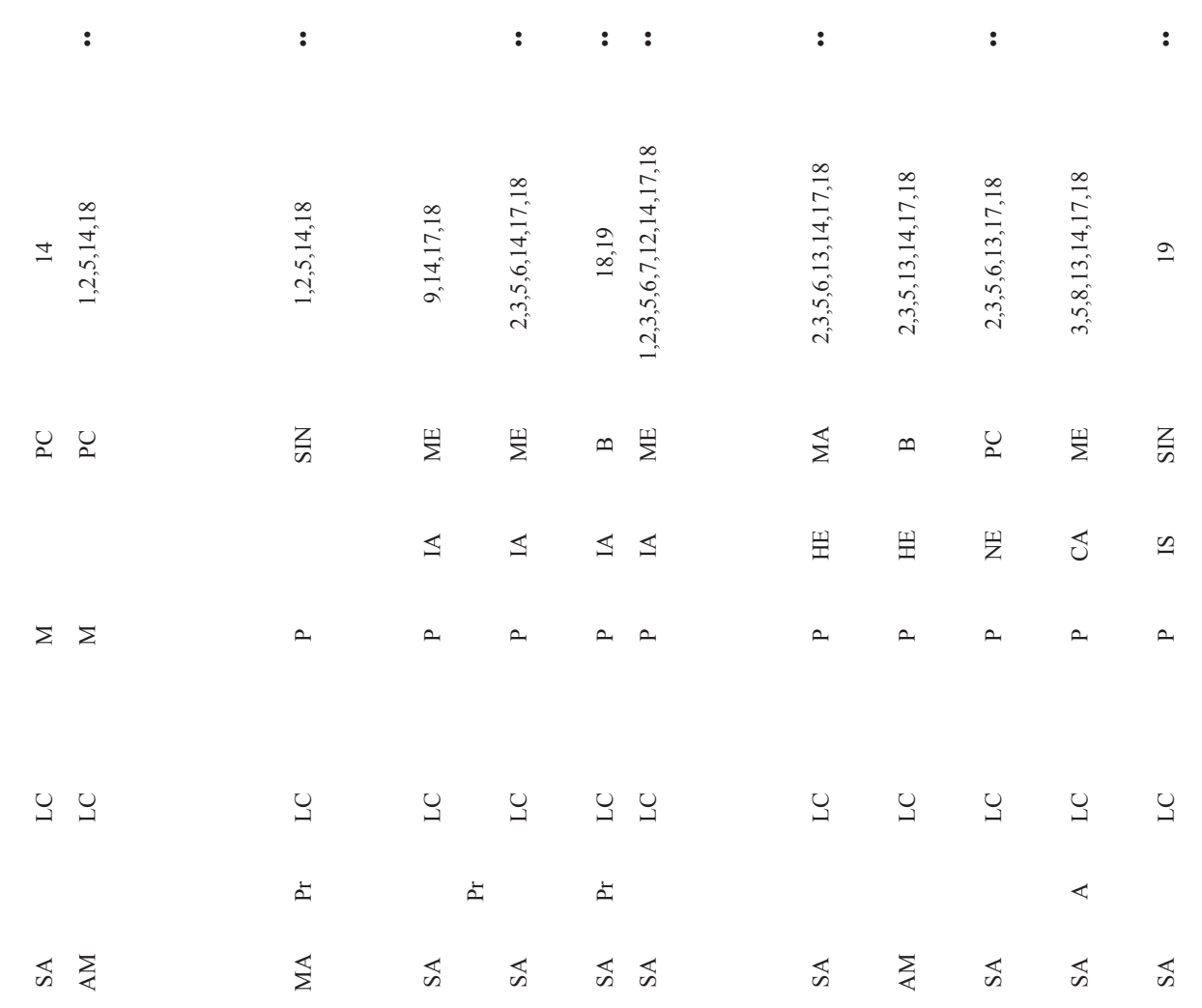

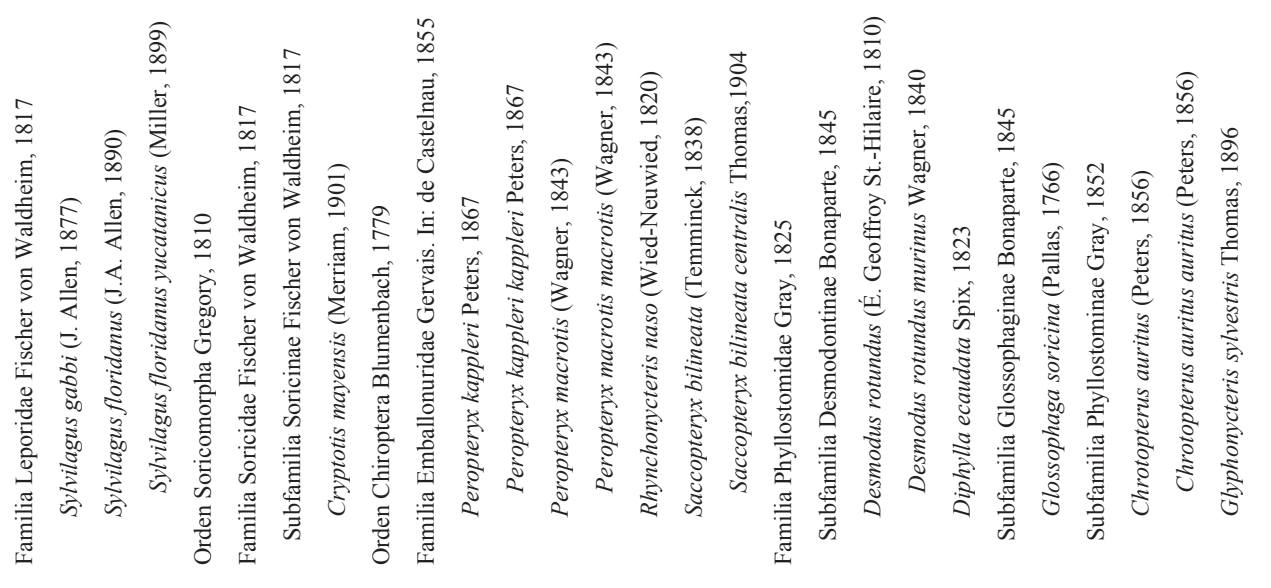




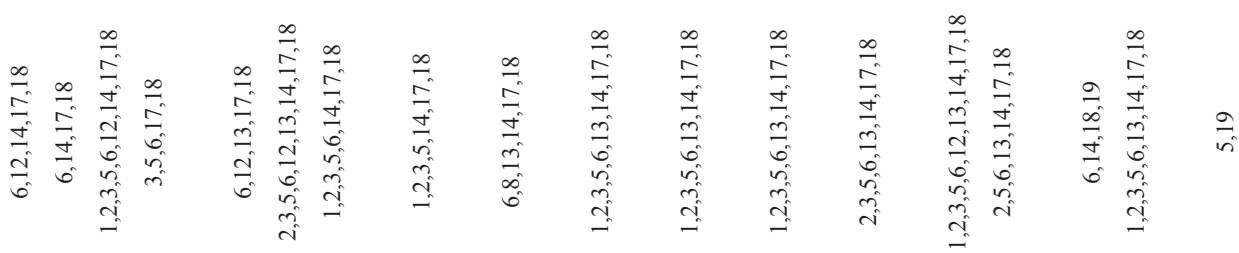

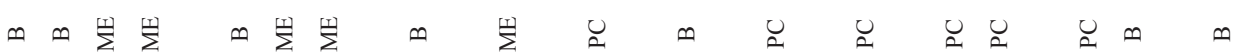

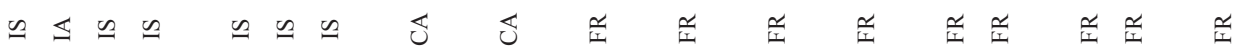

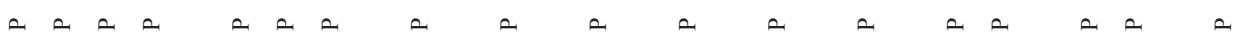

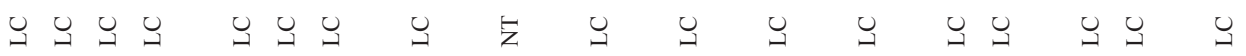
$\ll \ll \ll \quad \varangle \ll \ll \quad$ a

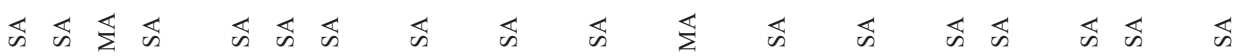

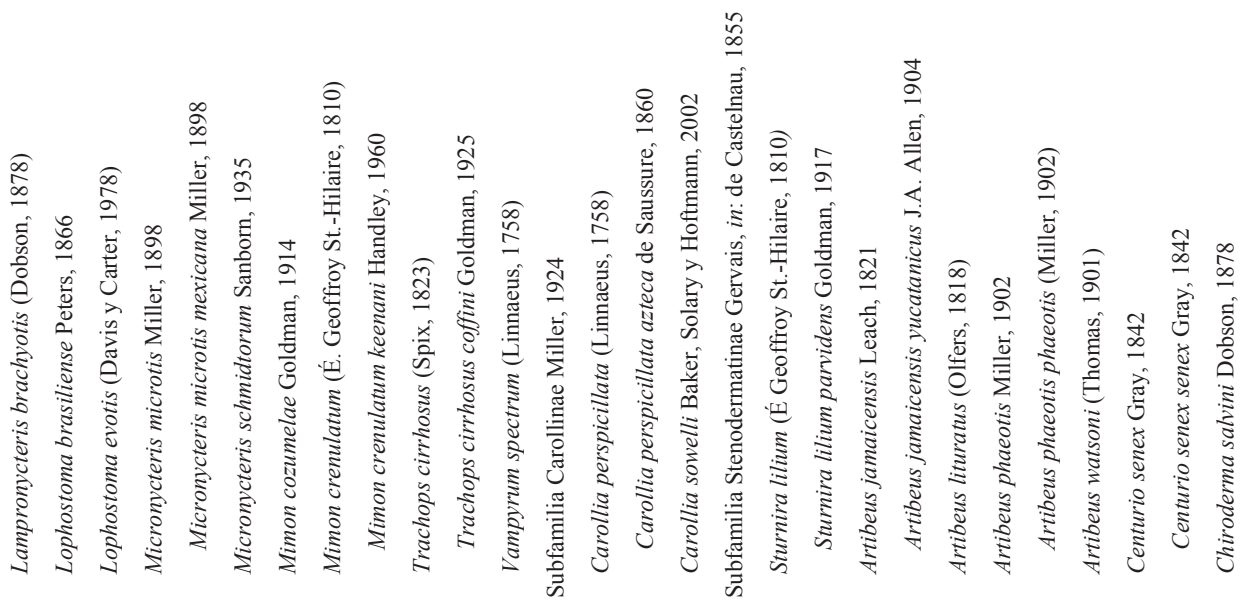




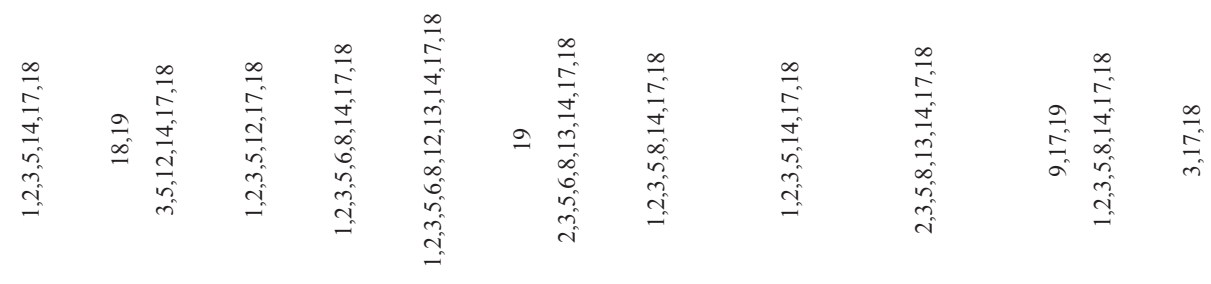

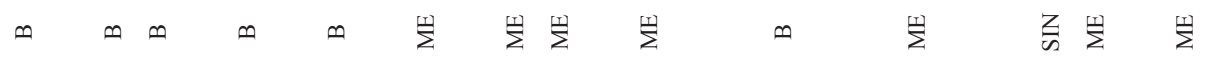

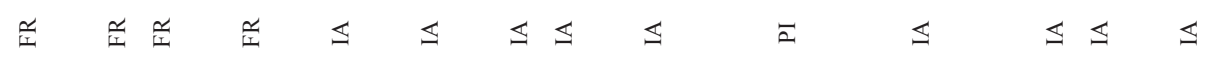

a a a a a a a a a a a a

U U U U U

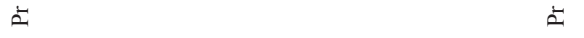

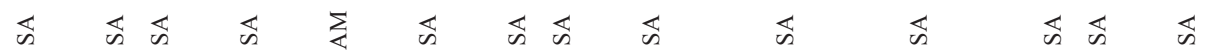

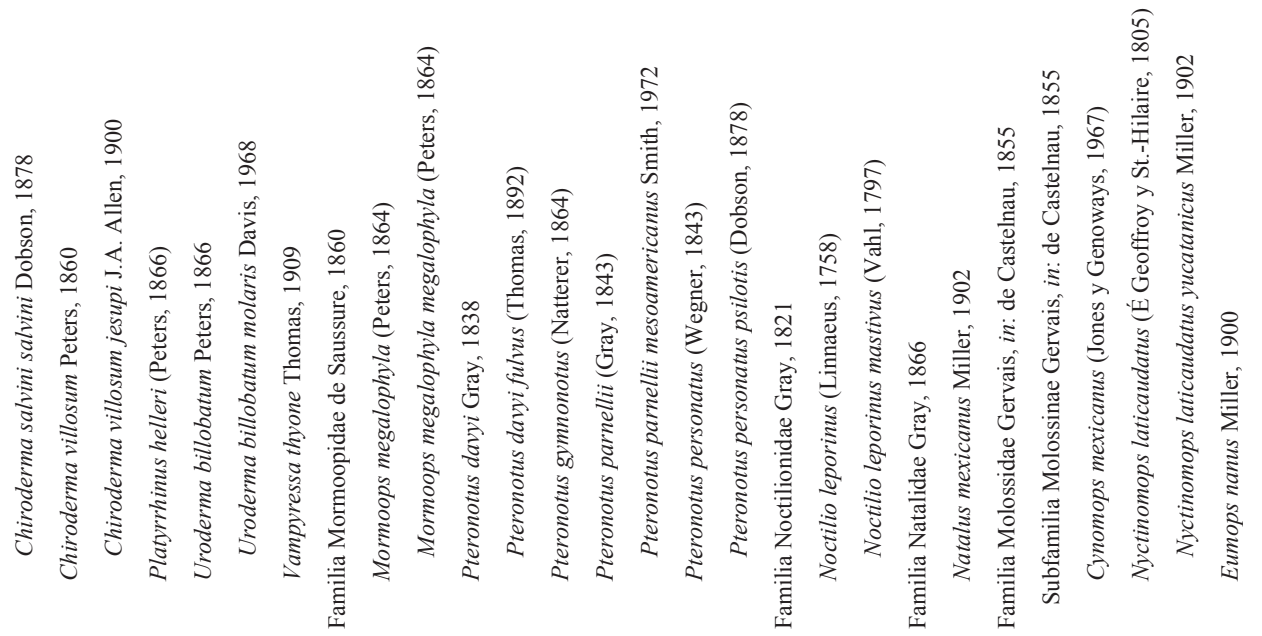




$$
\begin{aligned}
& \text { : } \quad: \quad: \quad:
\end{aligned}
$$

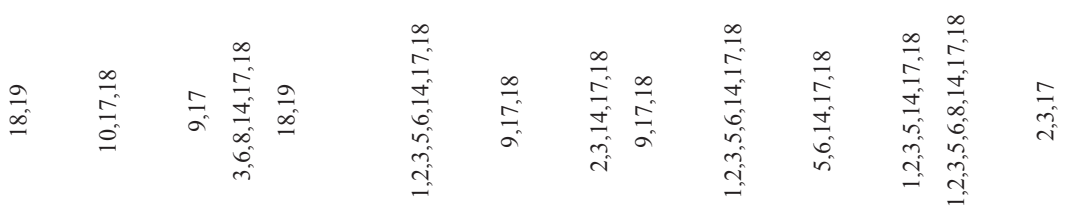

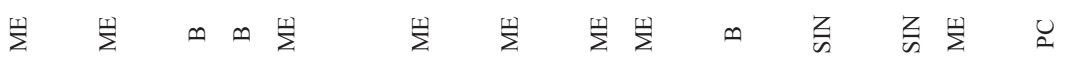

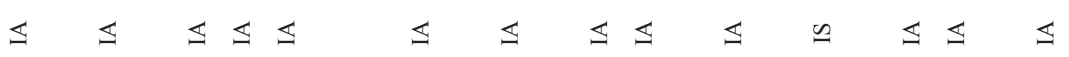

$$
\begin{aligned}
& a+a t a d a t a t a \\
& \text { ソ }
\end{aligned}
$$

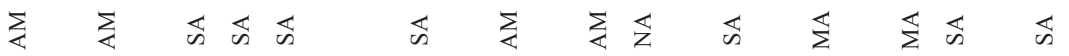

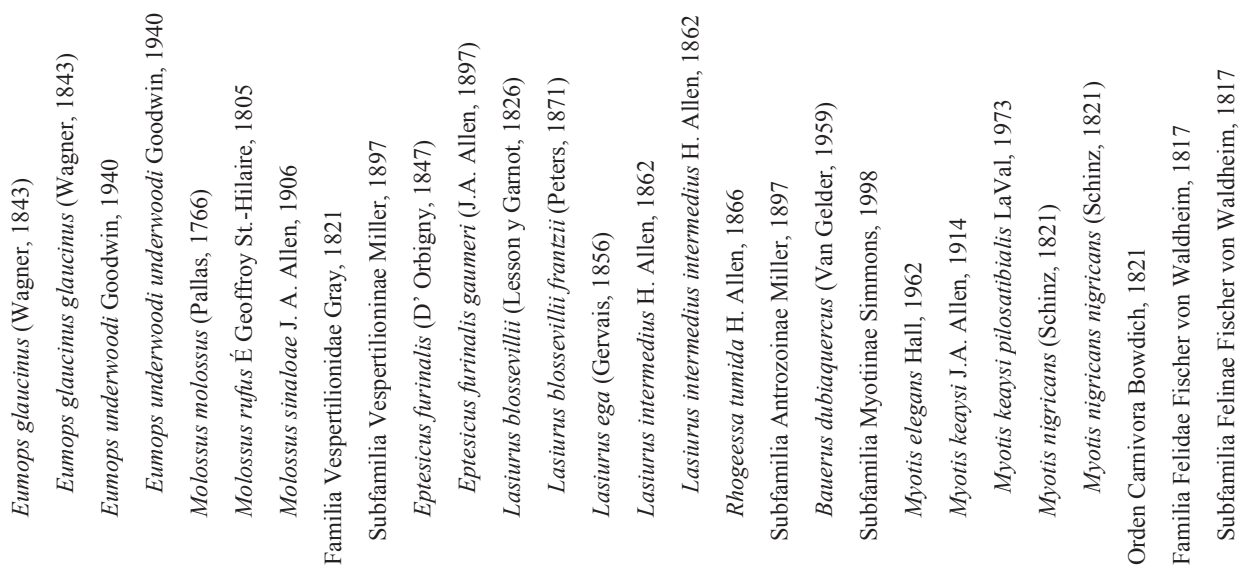




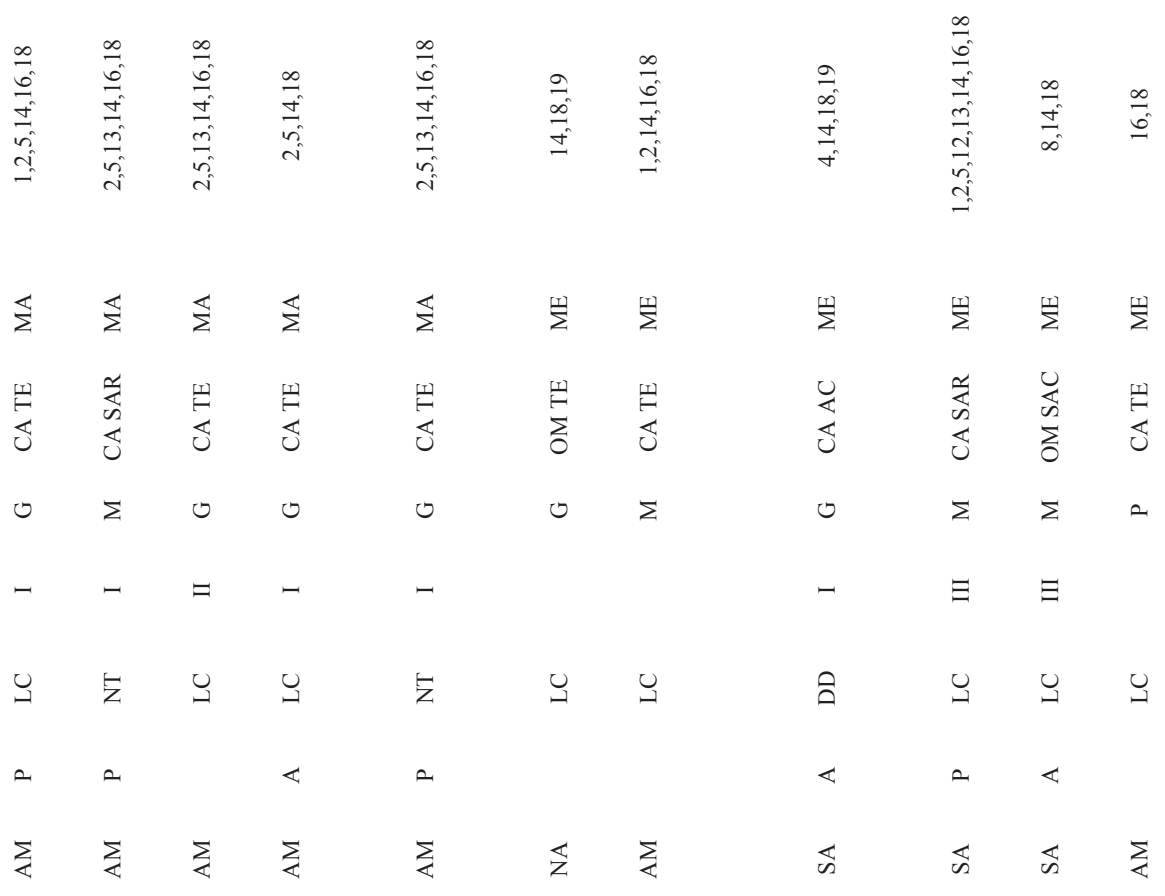

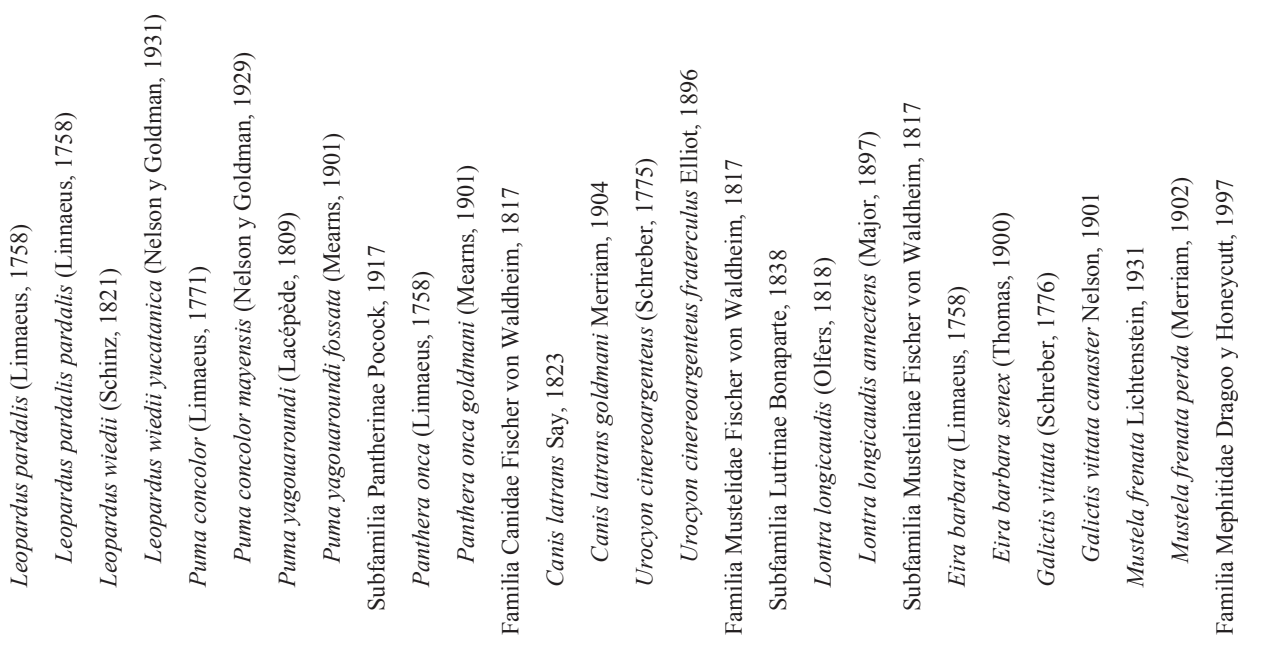




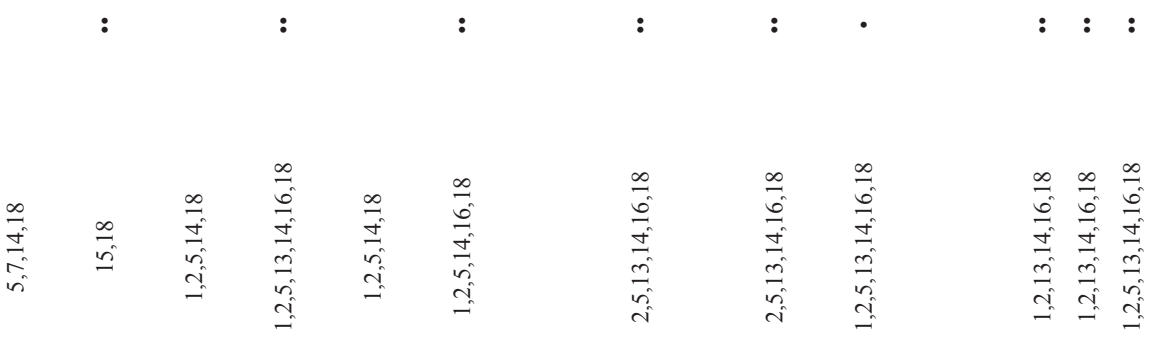

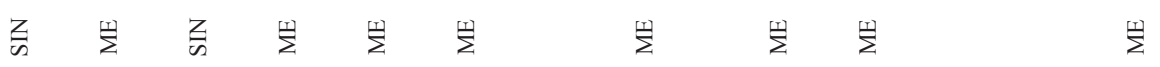

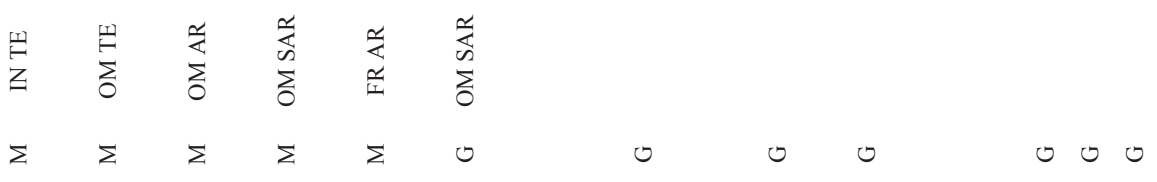

$$
\begin{aligned}
& \Xi \quad \exists \quad-\quad \text { ヨ }
\end{aligned}
$$

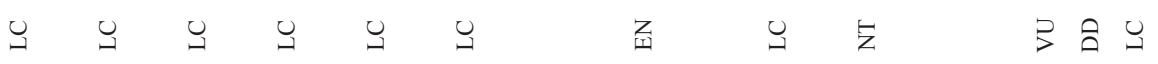

$$
\begin{aligned}
& \Rightarrow \quad 2 \quad 0 \quad a
\end{aligned}
$$

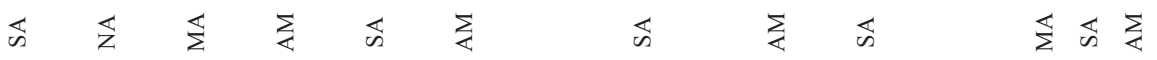

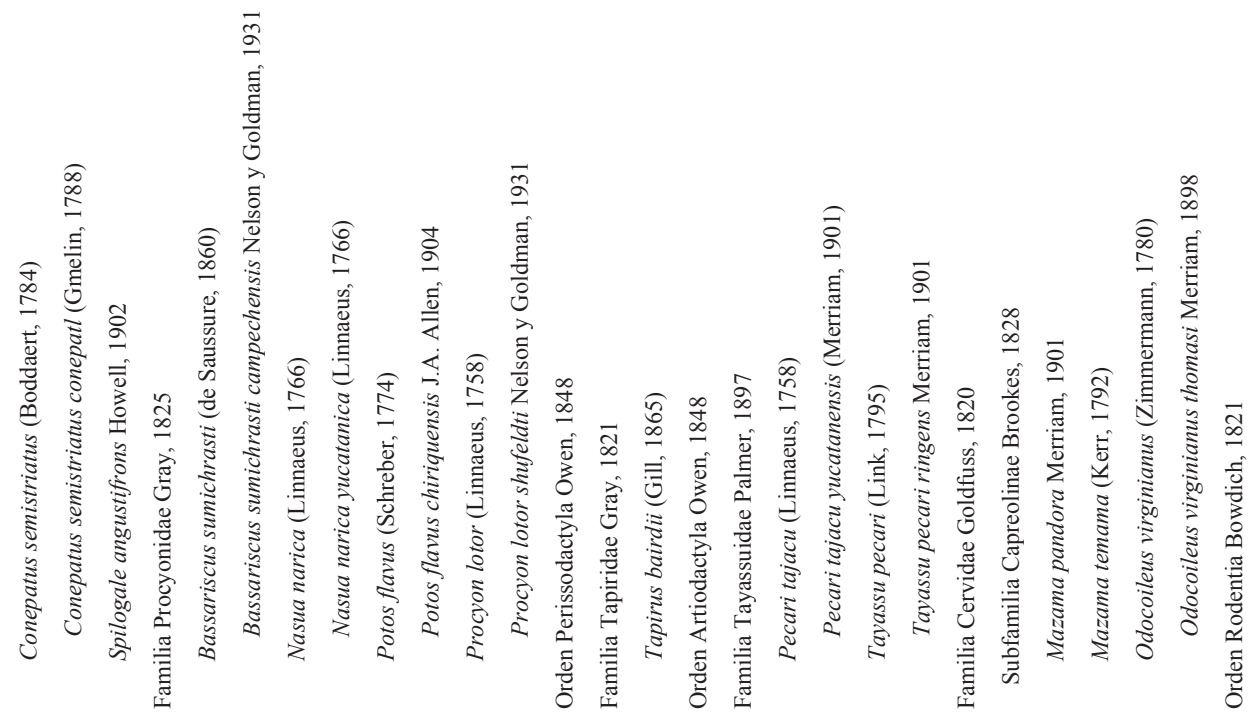




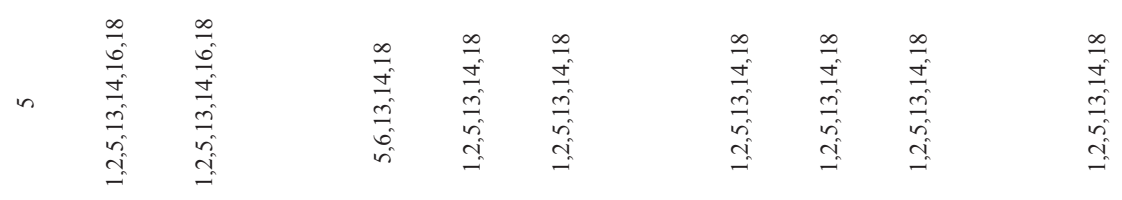

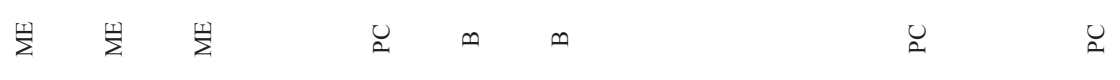

$a+a$
$\equiv$

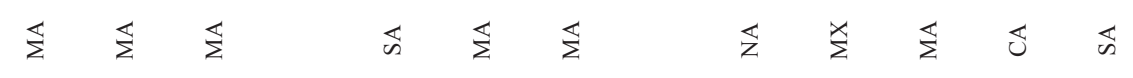

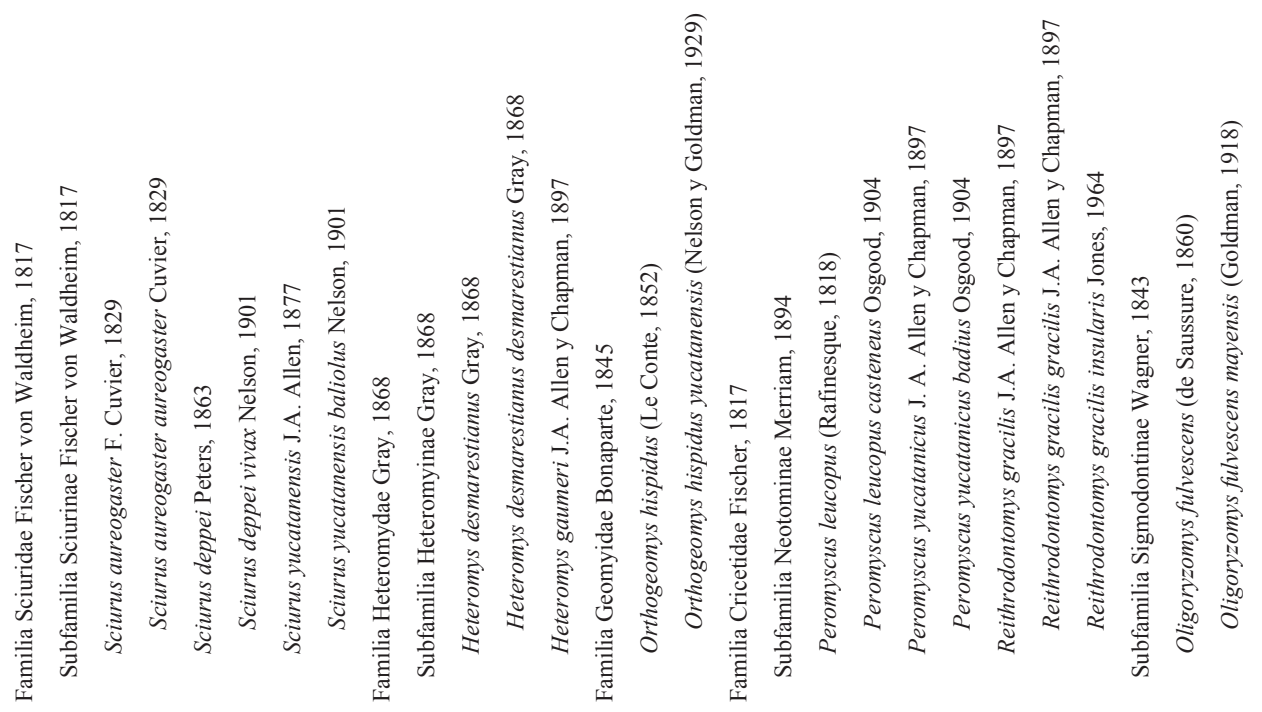


Guzmán-Soriano et al.: Mamiferos terrestres de Campeche

: : : : :

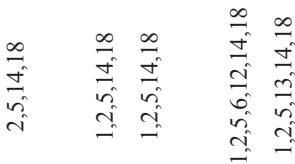

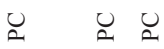

2

$2 a$

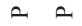

$\sum \sum \sum \sum$

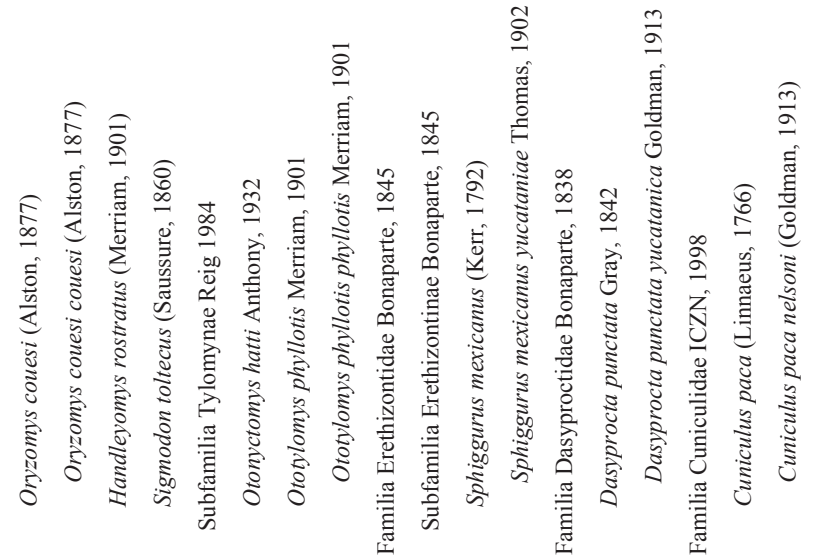

